\title{
Geometric and electronic structure of vanadium pentoxide: A density functional bulk and surface study
}

\author{
A. Chakrabarti, K. Hermann, R. Druzinic, M. Witko, ${ }^{*}$ F. Wagner, and M. Petersen \\ Theory Department, Fritz-Haber-Institut der MPG, Faradayweg 4-6, D-14195 Berlin, Germany
}

(Received 11 May 1998; revised manuscript received 23 October 1998)

\begin{abstract}
Density-functional theory (DFT) studies are performed to examine geometric and electronic properties of orthorhombic bulk $\mathrm{V}_{2} \mathrm{O}_{5}$ as well as of its (010) oriented surface. Electronic states, total energies, as well as atom forces (used to obtain equilibrium geometries) are computed with the $a b$ initio full-potential linear augmented plane wave method. The $\mathrm{V}_{2} \mathrm{O}_{5}(010)$ surface is modeled by periodic single layers in a repeated slab geometry, which is justified by the weak electronic interlayer coupling found in the bulk calculations. The electronic structure of the $\mathrm{V}_{2} \mathrm{O}_{5}(010)$ single-layer slabs, represented by their valence densities of states (DOS) and its atom contributions, is compared with results of bulk $\mathrm{V}_{2} \mathrm{O}_{5}$ and with previous results obtained by DFT surface cluster studies. The comparison yields good qualitative agreement between the different approaches, which confirms the local nature of interatomic binding in $\mathrm{V}_{2} \mathrm{O}_{5}$. Further, the computed valence DOS is used to interpret recent experimental results from photoemission on $\mathrm{V}_{2} \mathrm{O}_{5}(010)$, which suggests that differently coordinated oxygen sites at the surface can be identified in the spectrum. Thus, $\mathrm{V}_{2} \mathrm{O}_{5}(010)$ photoemission spectra may be used to monitor the participation of oxygen ions in respective surface reactions.
\end{abstract}

[S0163-1829(99)03616-4]

\section{INTRODUCTION}

Transition-metal oxides are noted for their rich and diverse structural, physical, and chemical properties. ${ }^{1-3}$ Among these, vanadium oxides represent an important class of systems that are widely studied and used in many technological applications. ${ }^{4,5}$ In particular, vanadium pentoxide, $\mathrm{V}_{2} \mathrm{O}_{5}$, or vanadia-based compounds from important catalysts for hydrocarbon oxidation. Further, $\mathrm{V}_{2} \mathrm{O}_{5}$ undergoes a reversible and fast transition from a semiconductor to a metal phase near $257^{\circ} \mathrm{C}$. Hence, it has potential applications for thermally activated electrical and optical switching devices and write-erase media. ${ }^{5}$ Despite the enormous importance of $\mathrm{V}_{2} \mathrm{O}_{5}$ as a catalyst many microscopic details of its catalytic behavior are still under debate, ${ }^{4}$ which makes a detailed study of $\mathrm{V}_{2} \mathrm{O}_{5}$ bulk and surface properties worthwhile.

So far, theoretical studies on $\mathrm{V}_{2} \mathrm{O}_{5}$ include those on its bulk structure using various semiempirical and $a b$ initio techniques. There are reports of pseudopotential periodic Hartree-Fock (HF) calculations ${ }^{6}$ to study the geometry and electronic structure. In this paper, restricted optimizations based on empirical force fields are performed and yield an equilibrium geometry close to the experimental result. Further, band structure and density of states (DOS) calculations give information on the electronic structure and partial ionicity of the oxide. The authors find a direct band gap at the $\Gamma$ point, which is highly overestimated $(12 \mathrm{eV})$ as expected from calculations using the HF approach. Results of a tightbinding study within a perturbative approach ${ }^{7}$ have been compared with various experimental spectroscopic data. The theoretical results are found to be in satisfactory agreement with $\mathrm{x}$-ray photoelectron and soft $\mathrm{x}$-ray spectroscopy as well as with electron energy-loss spectroscopic data. Further, the electronic structure of $\mathrm{V}_{2} \mathrm{O}_{5}$ has been examined by a nonempirical atomic orbital method, ${ }^{8}$ by $a b$ initio orthogonalized linear combination of atomic orbitals studies, ${ }^{9}$ by selfconsistent augmented spherical wave calculations ${ }^{10,11}$ using the density-functional theory (DFT) scheme within the linear density approximation (LDA), ${ }^{12}$ and by DFT pseudopotential $^{13}$ studies. These studies yield overall good agreement with the measured photoemission and optical properties. Further, calculations on $\mathrm{V}_{2} \mathrm{O}_{5}$ using a selfconsistent field crystal orbital formalism based on the intermediate neglect of differential overlap ${ }^{14}$ together with infrared spectroscopic measurements have been reported in the literature. These studies suggest that oxygen vacancies of highest coordination are most likely to appear in oxidation processes at $\mathrm{V}_{2} \mathrm{O}_{5}$ surfaces. Moreover, there are recent reports of size converged density functional theory (DFT) cluster calculations ${ }^{15-18}$ on electronic properties at the $\mathrm{V}_{2} \mathrm{O}_{5}(010)$ surface in order to understand its catalytic activity. These calculations establish the mixed ionic/covalent binding character of $\mathrm{V}_{2} \mathrm{O}_{5}$ and show an increased local surface reactivity of the two and three fold coordinated bridging oxygen species compared to terminal vanadyl oxygen. This is confirmed by results from an electrostatic potential analysis based on the cluster charge distribution ${ }^{15}$ as well as by hydrogen adsorption studies. ${ }^{16}$

Recent developments of highly efficient and accurate $a b$ initio algorithms make reliable electronic structure calculations and geometry optimizations of very complex transitionmetal oxides feasible. In the present paper, we report results from calculations of the geometric and electronic structure of $\mathrm{V}_{2} \mathrm{O}_{5}$ within the DFT approach using the $a b$ initio fullpotential linear augmented plane-wave method. ${ }^{19-21}$ In addition to bulk calculations, we study periodic layer slabs of (010) orientation in a repeated slab geometry. The computational results show altogether that the electronic binding between adjacent (010) slabs forming physical building blocks of the (layer-type) $\mathrm{V}_{2} \mathrm{O}_{5}$ bulk is rather weak. This justifies 


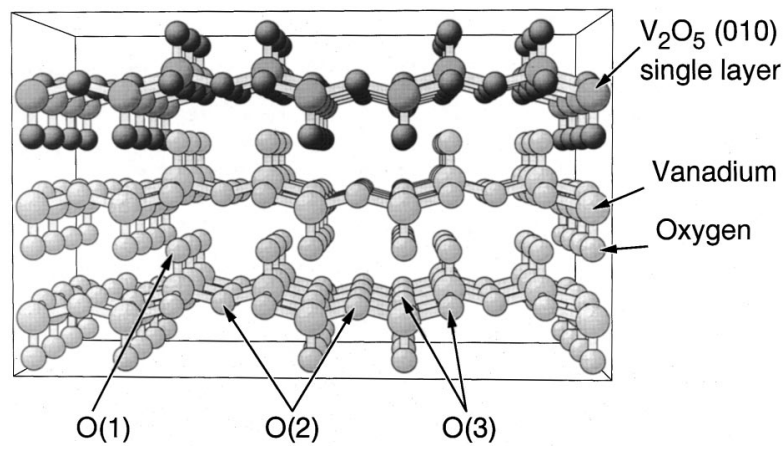

FIG. 1. Crystal section of orthorhombic $\mathrm{V}_{2} \mathrm{O}_{5}$ with netplane stacking along the (010) direction. Vanadium and inequivalent oxygen centers, singly coordinated $\mathrm{O}(1)$, doubly coordinated $\mathrm{O}(2)$, and triply coordinated $\mathrm{O}(3)$, are marked accordingly. All atoms of the $\mathrm{V}_{2} \mathrm{O}_{5}(010)$ single-layer slab at the top are emphasized by darker shaded balls.

the use of these slabs (called "single-layer slabs" in the following) in modeling the electronic structure at the bulk terminated (010) surface of $\mathrm{V}_{2} \mathrm{O}_{5}$. The valence electronic structure of $\mathrm{V}_{2} \mathrm{O}_{5}(010)$ single-layer slabs, represented by their valence DOS, is compared with results of previous DFT surface cluster model calculations $\mathrm{s}^{22}$ and yields good qualitative agreement. This suggests strongly that embedded clusters as small as $\mathrm{V}_{10} \mathrm{O}_{31} \mathrm{H}_{12}$ (Ref. 15) (referring to small single-layer sections) can provide meaningful models of local environments at the $\mathrm{V}_{2} \mathrm{O}_{5}(010)$ surface. The computed theoretical DOS of the $\mathrm{V}_{2} \mathrm{O}_{5}(010)$ single-layer slabs are compared with recent angle-resolved ultraviolet photoemission spectroscopy (ARUPS) data ${ }^{22}$ and leads to a microscopic interpretation of the experiment, which may be used to give information about the involvement of differently coordinated oxygen sites in surface reactions.

In Sec. II, we describe briefly computational details and Sec. III presents results and discussion. Finally, we summarize our conclusions in Sec. IV.

\section{COMPUTATIONAL METHODS}

Bulk vanadium pentoxide $\mathrm{V}_{2} \mathrm{O}_{5}$ forms an orthorhombic (Pmmn) layer crystal $^{15,23,24}$ with lattice parameters $a=11.51, b=4.37$, and $c=3.56 \AA$. The physical layers with normal direction along (010) (Ref. 25) are composed of distorted $\mathrm{VO}_{5}$ pyramids sharing edges and corners, see Fig. 1, with their elementary cell containing two element units (2 $\times \mathrm{V}_{2} \mathrm{O}_{5}, 14$ atoms) and six planar atom layers (4 oxygen layers, 2 vanadium layers). In the following we use the term "single-layer slab" to denote these physical layers. There are three structurally inequivalent oxygen centers in the $\mathrm{V}_{2} \mathrm{O}_{5}$ compound, terminal (vanadyl) oxygen, $\mathrm{O}(1)$, oxygen bridging two vanadium centers, $\mathrm{O}(2)$, and bridging oxygen coordinated to three vanadium centers, $\mathrm{O}(3)$.

The detailed geometry of bulk $\mathrm{V}_{2} \mathrm{O}_{5}$ is obtained in the present calculations from a total energy optimization where the experimental structure (orthorhombic symmetry) ${ }^{23}$ serves as a starting point. The optimization is performed in two steps. First, optimized lattice constants, $a, b, c$, are determined by lattice scaling such that the total energy of the bulk unit cell is smallest. Second, the positions of all 14 atoms in the optimized unit cell are allowed to relax according to the force field (computed from total energy gradients) acting on the corresponding nuclei. The bulk system is considered to be at equilibrium if all force components are smaller than 0.5 $\mathrm{mRy} / \mathrm{Bohr}$. Ideally, the present two-step optimization has to be repeated iteratively until geometric self-consistency is achieved. However, the non-self-consistent optimization procedure has been tested in its accuracy and applied successfully to numerous bulk systems, see e.g., Ref. 26. It is adopted in the present calculations for computational convenience and is substantiated by the good agreement between theoretical and experimental geometries described in Sec. III A.

In addition to the optimized theoretical bulk structure of $\mathrm{V}_{2} \mathrm{O}_{5}$, we consider also an idealized bulk geometry that has been applied extensively in previous cluster work. ${ }^{15,16,27,28}$ Here, the $\mathrm{VO}_{5}$ pyramids of the (010) layer are idealized by using averaged interatomic $\mathrm{V}$-O distances $d_{\mathrm{V}-\mathrm{O}}=1.89 \AA$ and $\mathrm{O}-\mathrm{V}-\mathrm{O}$ angles, $86.18^{\circ}$, for all bridging oxygen centers. Further, terminal vanadyl oxygen centers are assumed directly on top of vanadium centers in the direction normal to the (010) layer with $d_{\mathrm{V}-\mathrm{O}}=1.58 \AA$. This results in local $\mathrm{VO}_{5}$ pyramids with square base and fourfold rotation symmetry in addition to the inversion, twofold rotation, and mirror symmetries of the (010) layers, which reduces the computational effort of the cluster calculations.

In order to study the importance of interlayer coupling in bulk $\mathrm{V}_{2} \mathrm{O}_{5}$ additional studies are performed on the electronic structure of separated two-dimensional periodic $\mathrm{V}_{2} \mathrm{O}_{5}(010)$ single-layer slabs, see Fig. 1. The calculations are based on a repeated slab geometry where the layers are repeated along the (010) netplane direction with a large interlayer distance $\Delta z_{(010)}$. Test calculations with varying $\Delta z_{(010)}$ have shown that a value $\Delta z_{(010)}=10.8 \AA$ (corresponding to $2.5 b$ with $b$ denoting the respective bulk lattice constant) is sufficient to guarantee electronic separation between adjacent single-layer slabs. An increase of $\Delta z_{(010)}$ from 2.5 to $2.8 b$ results in a difference of only $0.016 \mathrm{Ry}(0.2 \mathrm{eV})$ of the unit cell total energy (amounting to $-9030.623 \mathrm{Ry}$ ). For the separation $\Delta z_{(010)}=2.5 b$ the positions of all atoms inside the (010) layer unit cell are reoptimized analogous to the bulk procedure.

The electronic structure of the $\mathrm{V}_{2} \mathrm{O}_{5}$ bulk and slab systems is calculated within the density-functional theory (DFT) formalism using the local-density approximation ${ }^{12}$ (LDA) as well as gradient corrected functionals ${ }^{29}$ (GGA-II) for exchange and correlation. Comparative studies on bulk $\mathrm{V}_{2} \mathrm{O}_{5}$ with both approximate functionals show rather small differences that do not affect the present conclusions. Therefore, in the following only results from calculations within the LDA scheme will be discussed. Total energies and derived quantities are obtained with the $a b$ initio full potential linearized augmented plane-wave (FP-LAPW) method. ${ }^{19-21}$ Extended test calculations have proven to yield sufficient accuracy in the total energy minimization of bulk $\mathrm{V}_{2} \mathrm{O}_{5}$ using two $k$ points inside the irreducible part of the Brillouin zone, an energy cut-off for plane-wave expansions in the interstitial region of $22 \mathrm{Ry}$, and $l_{\max }=10$ for the upper limit of the angular momentum expansions of the wave functions inside the atom spheres. Respective densities of states (DOS) are evaluated with $48 k$ points inside the irreducible part of the 
TABLE I. Comparison of the orthorhombic lattice constants, $a, b, c$, of bulk $\mathrm{V}_{2} \mathrm{O}_{5}$ obtained from the present optimization with values from two different experimental analyses (Refs. 23 and 24). All values are given in $\AA$.

\begin{tabular}{ccc}
\hline \hline $\begin{array}{l}\text { Lattice } \\
\text { constant }\end{array}$ & This work & \multicolumn{2}{c}{ Experimental } \\
\hline$a$ & 11.375 & 11.519 (Ref. 23), 11.512 (Ref. 24) \\
$b$ & 4.318 & 4.373 (Ref. 23), 4.368 (Ref. 24) \\
$c$ & 3.519 & 3.564 (Ref. 23), 3.564 (Ref. 24) \\
\hline \hline
\end{tabular}

orthorhombic Brillouin zone while the detailed bulk band structure is based on $81 k$ points along high-symmetry directions. In the repeated slab calculations of the $\mathrm{V}_{2} \mathrm{O}_{5}(010)$ single-layer system all numerical parameters are taken from the bulk study except for the energy cut off, which is reduced to $18.5 \mathrm{Ry}$ as a compromise between numerical accuracy and computational effort. Test calculations on bulk $\mathrm{V}_{2} \mathrm{O}_{5}$ with cut-off values of 18.5 and 22 Ry yielded relative total energy differences of only $5 \times 10^{-5}$ with an increase in computer time of $50 \%$. The structure optimizations of the present systems are performed using the damped Newton dynamics method. $^{20,30}$ This approach has been verified by an alternative minimization scheme using the variable metric method. ${ }^{20,21,31}$

The electronic structure results of the FP-LAPW calculations on the periodic bulk and slab systems are compared with those of earlier DFT surface cluster studies on wellconverged clusters as large as $\mathrm{V}_{16} \mathrm{O}_{49} \mathrm{H}_{18}$ representing local environments at the $\mathrm{V}_{2} \mathrm{O}_{5}(010)$ surface. Technical details on the cluster work can be found in Refs. 15 and 22 .

\section{RESULTS AND DISCUSSION}

\section{A. Geometric structure}

The computed lattice constants for orthorhombic bulk $\mathrm{V}_{2} \mathrm{O}_{5}$ are listed in Table I together with data from two different experimental analyses. ${ }^{23,24}$ A comparison shows that the calculated values are smaller by only $1.25 \%$ with respect to experiment. The decreased theoretical values are partially due to the local-density approximation for exchange and correlation used in the present work, which is generally known to yield lattice constants which are smaller by $2-6 \%$ with respect to experiment. ${ }^{32}$ Table II compares the computed

TABLE II. Optimized positions of all inequivalent atom centers inside the periodicity cell of orthorhombic bulk $\mathrm{V}_{2} \mathrm{O}_{5}$ and of the $\mathrm{V}_{2} \mathrm{O}_{5}$ (010) single-layer slab, see text. The number of symmetry equivalents of each atom are given in square brackets. For a definition of $\mathbf{r}_{\exp }=\left(x_{\exp }, y_{\exp }, z_{\exp }\right)$ see text. Quantities $\left|\Delta r_{\text {bulk }}\right|$ and $\left|\Delta r_{\text {slab }}\right|$ refer to distances between the experimental bulk positions and those of the present optimizations. All length values are given in $\AA$.

\begin{tabular}{lccc}
\hline \hline Atom & $\left|\Delta r_{\text {bulk }}\right|$ & $\left|\Delta r_{\text {slab }}\right|$ & $\mathbf{r}_{\text {exp }}$ \\
\hline$V[\times 4]$ & 0.013 & 0.013 & $10.233,0.469,0.880$ \\
$O(1)[\times 4]$ & 0.002 & 0.026 & $10.192,2.035,0.880$ \\
$O(2)[\times 2]$ & 0.001 & 0.053 & $8.531,-0.013,0.880$ \\
$O(3)[\times 4]$ & 0.013 & 0.029 & $10.589,0.011,2.640$ \\
\hline \hline
\end{tabular}

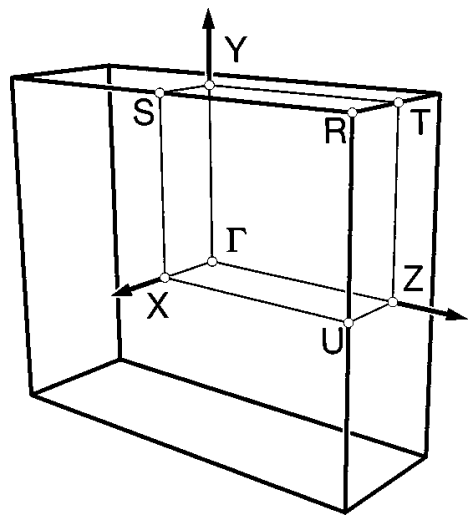

FIG. 2. Brillouin zone of the orthorhombic $\mathrm{V}_{2} \mathrm{O}_{5}$ lattice with high-symmetry points labeled accordingly. Note that the layer normal direction corresponds to $\Gamma Y$ in this plot.

equilibrium positions of all inequivalent atoms of $\mathrm{V}_{2} \mathrm{O}_{5}$ with those from bulk experiments. ${ }^{23}$ Here, the measured positions, given by cartesian coordinates $\mathbf{r}_{\exp }=\left(x_{\exp }, y_{\exp }, z_{\exp }\right)$, have been scaled by a factor 0.9875 to bring the experimental and theoretical unit cell volumes in exact agreement. The distances between the experimental atom positions and those of the present bulk optimization, quantified by $\left|\Delta r_{\text {bulk }}\right|$ in Table II, are in all cases smaller or close to $0.01 \AA$, which confirms the excellent agreement of the theoretical structure with experiment.

Results from the geometry optimization of separated $\mathrm{V}_{2} \mathrm{O}_{5}(010)$ single-layer slabs are also included in Table II. Here, the computed equilibrium positions of all inequivalent slab atoms are compared with those of the $\mathrm{V}_{2} \mathrm{O}_{5}$ bulk optimization and with experiment. ${ }^{23}$ Analogous to the bulk discussion, $\left|\Delta r_{\text {slab }}\right|$ defines, for each atom, the distance between its experimental position and that of the slab optimization. A comparison of the $\left|\Delta r_{\text {slab }}\right|$ with the $\left|\Delta r_{\text {bulk }}\right|$ values reveals extremely small differences. Thus, the optimized structure of the separated $\mathrm{V}_{2} \mathrm{O}_{5}(010)$ single-layer slab reproduces that of a single layer slab inside bulk $\mathrm{V}_{2} \mathrm{O}_{5}$. This close geometric similarity shows that electronic interlayer coupling is of minor importance for the present oxide. Further, it suggests strongly that the geometry and electronic structure of the $\mathrm{V}_{2} \mathrm{O}_{5}(010)$ surface (terminated by a single-layer slab) can be represented quite well by that of a $\mathrm{V}_{2} \mathrm{O}_{5}(010)$ single-layer slab. Hence, the present theoretical results are consistent with the absence of major reconstruction at the clean single crystal $\mathrm{V}_{2} \mathrm{O}_{5}(010)$ surface, which is observed in the experiment published so far. ${ }^{33,34}$ However, it should be noted that the present optimization procedure based on energy gradients and starting from bulk equilibrium cannot rule out alternative reconstructed geometries that are separated from the present structure by energy barriers.

\section{B. Electronic structure}

\section{$\mathrm{V}_{2} \mathrm{O}_{5}$ bulk calculations}

The electronic structure of the three-dimensional periodic $\mathrm{V}_{2} \mathrm{O}_{5}$ bulk is characterized by energy bands and band states inside the irreducible part of the orthorhombic Brillouin zone (BZ) as sketched in Fig. 2. The left part of Fig. 3 shows the calculated band structure for the optimized $\mathrm{V}_{2} \mathrm{O}_{5}$ bulk geom- 

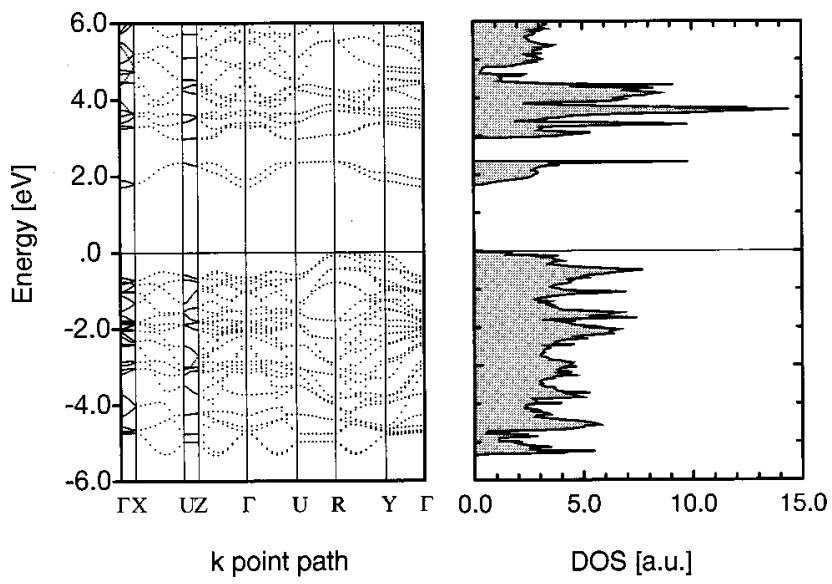

FIG. 3. Band structure and total DOS of bulk $\mathrm{V}_{2} \mathrm{O}_{5}$. The energy bands are shown for characteristic paths connecting high-symmetry points of the irreducible part of the Brillouin zone. For a definition of the high-symmetry points see Fig. 2. All energies $\varepsilon(k)$ are taken with respect to the Fermi energy. The DOS is given in states per unit cell and per eV.

etry where energy bands $\varepsilon(k)$ are given for characteristic paths connecting high-symmetry points of the BZ. All energy values are taken with respect to the Fermi level such that negative energies refer to valence bands while positive values characterize conduction bands. The right part of Fig. 3 displays the total density of states (DOS) where no energy broadening is applied to the DOS. From photodesorption experiments $\mathrm{V}_{2} \mathrm{O}_{5}$ is known to be a semiconductor with a visible band gap of $E_{g}=2.35 \mathrm{eV}$ (Ref. 35) at low temperatures. This is confirmed by values obtained from other measurements, for example, optical absorption $\left(E_{g}=2.30 \mathrm{eV}\right)$ (Ref. 36) and optical reflectance measurements $\left(E_{g}=2.38 \mathrm{eV}\right) .^{37}$ The present calculations yield a direct band gap of $2.3 \mathrm{eV}$ at $\Gamma$ and an indirect gap (corresponding to a transition $R$ to $\Gamma$, see Fig. 3) of $1.9 \mathrm{eV}$, which seems to be in good agreement with the experimental gap values. However, this agreement has to be considered fortuitous since band-structure methods based on the DFT approach, such as FP-LAPW, refer to ground state properties and are not expected to describe electronic excitations to a high accuracy. As a general result, optical gaps of bulk materials measured by absorption experiments are underestimated in DFT-based models. ${ }^{38}$

There are two narrow split-off conduction bands at about $2 \mathrm{eV}$ above the Fermi level and about $0.5 \mathrm{eV}$ below the upper conduction-band range, which have been discussed previously in the literature..$^{9,11,39}$ These bands are characterized by rather small dispersion widths corresponding to large effective electron masses and localized band states. The latter are described by dominant V $3 d_{x y}$ character $\left(t_{2 g}\right.$ symmetry) with small $\mathrm{O} 2 p_{x, y}$ admixing. The presence of these split-off conduction bands hints at possible excitations to localized electron states and is of interest for conductivity as well as optical absorption experiments on bulk $\mathrm{V}_{2} \mathrm{O}_{5}$. ${ }^{9,11,39}$

The energy region of the calculated $\mathrm{V}_{2} \mathrm{O}_{5}$ valence bands is found to have a width of $5.5 \pm 0.5 \mathrm{eV}$. This matches very well with values obtained from angle-integrated ${ }^{40}$ as well as angle-resolved ultraviolet photoemission spectroscopy ${ }^{22,41}$ on $\mathrm{V}_{2} \mathrm{O}_{5}$. As an example, Fig. 4 shows very recent ARUPS data for single crystal $\mathrm{V}_{2} \mathrm{O}_{5}$ using He-II light at normal inci-

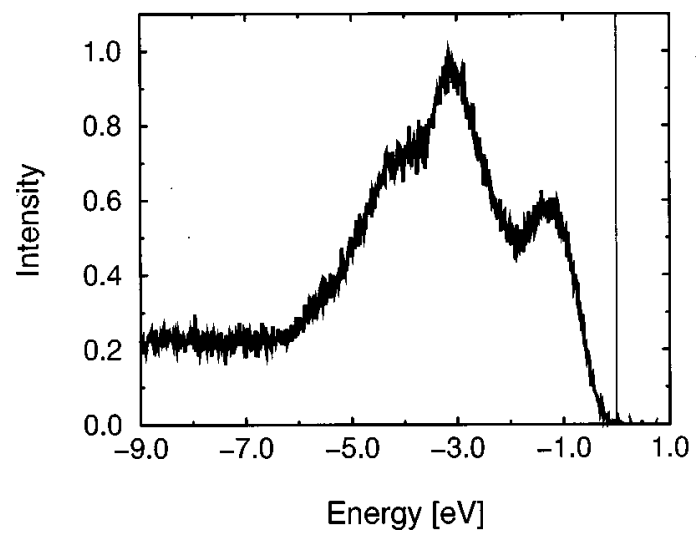

FIG. 4. Angle resolved He-II ultraviolet photoemission spectrum of a $\mathrm{V}_{2} \mathrm{O}_{5}(010)$ surface sample taken at normal incidence (Refs. 22 and 41).

dence with respect to the $(010)$ oriented surface. ${ }^{22,41}$ The emission spectrum has an overall width of $6 \mathrm{eV}$ and can be described by a superposition of mainly three peaks, a major central peak at about $3.2 \mathrm{eV}$ below the onset of the spectrum (taken as the energy zero in Fig. 4), and two satellite peaks at 1.2 and $4.3 \mathrm{eV}$, respectively. The origins of these peaks can be identified by the present theory assuming that the dominant contributions to the emission intensity come from energy variations of the $\mathrm{V}_{2} \mathrm{O}_{5}$ valence density of states (and its atom projections) while the energy dependence of respective transition matrix elements is small within this energy region.

Figure 5 shows a decomposition of the calculated valence DOS for bulk $\mathrm{V}_{2} \mathrm{O}_{5}$ into atom projected contributions (partial density of states, PDOS). The projections refer to atom charge contributions of the band states within respective muffin-tin spheres about the inequivalent atom centers inside the elementary cell. Further, the PDOS curves have been smoothed by Gaussian broadening (of $0.5 \mathrm{eV}$ width, reflecting the experimental resolution in Fig. 4). The curve denoted "summed DOS'" in Fig. 5 represents the sum of all atom contributions inside the muffin-tin spheres. The multipeak structure of the summed DOS curve reflects the valence-band region that is dominated by $\mathrm{O} 2 p$-derived band states with some $\mathrm{V} 3 d$ contributions. It is only remotely similar to the three-peak structure observed in the photoemission experiment $^{41}$ and shown in Fig. 4. However, its atom decom-

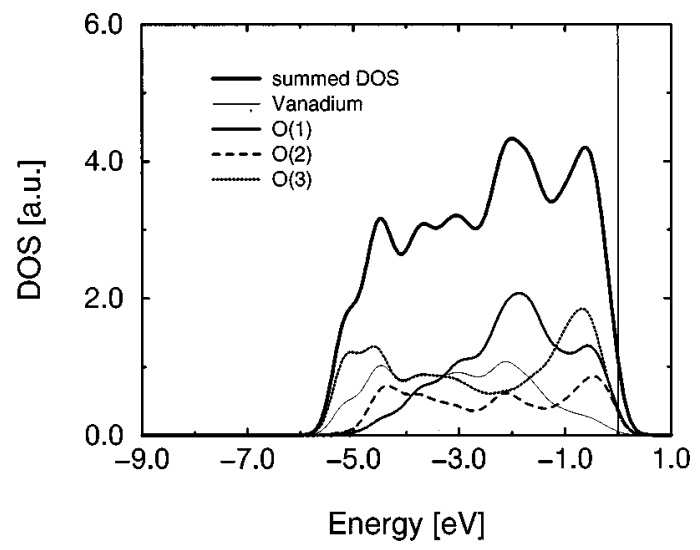

FIG. 5. Atom projected PDOS contributions for bulk $\mathrm{V}_{2} \mathrm{O}_{5}$. All values are given in states per unit cell and per $\mathrm{eV}$. 
position is very instructive. The PDOS due to terminal oxygen, $\mathrm{O}(1), 2 p$ states is peaked near $-2 \mathrm{eV}$, close to the center of the valence region, with a higher energy tail and is energetically more confined than the PDOS curves of the other oxygen ions. The latter extend over the complete valence region between -6 and $0 \mathrm{eV}$ with contributions that are higher at the valence periphery than near the center. This is particularly obvious for the PDOS of triply coordinated oxygen $\mathrm{O}(3)$, which dominates the low- and high-energy side of the valence-band region while the PDOS of doubly coordinated oxygen $\mathrm{O}(2)$ shows smaller variations. The $\mathrm{V}$ $3 d$-derived band states hybridize with $\mathrm{O} 2 p$-functions to form $\mathrm{V}-\mathrm{O}$ bonds in the system and contribute to the valence DOS in the lower part of the valence region. The energy dependence of these metal derived PDOS contributions is reasonable whereas their relative size may be somewhat too large compared to those of oxygen contributions. This is due to the projection method used to evaluate the PDOS's where metal centers with large muffin-tin radii are weighted more strongly than oxygen centers. Altogether, the present analysis suggests that the experimental photoemission spectrum shown in Fig. 4, reflecting the valence-band region of $\mathrm{V}_{2} \mathrm{O}_{5}$, can be interpreted by the energetic distribution of the different $\mathrm{O} 2 p$ valence-band states. The contributions from differently coordinated oxygen ions are energetically separated in the spectrum where the central valence region (main peak of Fig. 4) is dominated by singly coordinated oxygen $\mathrm{O}(1)$, while higher coordination oxygen $[\mathrm{O}(2,3)]$ contributions dominate the periphery. It should be mentioned that earlier semiempirical calculations ${ }^{7,14}$ predict an energetic order of the oxygen contributions which differs from that of the present calculations. These authors find $\mathrm{O}(1)$ derived states being located close to the Fermi level whereas $\mathrm{O}(2,3)$ states dominate the bottom region of the valence band.

In order to characterize the electronic structure and local charging in bulk $\mathrm{V}_{2} \mathrm{O}_{5}$ in greater detail, the spatial distribution of valence electrons inside the elementary unit cell is examined. Contour maps of the difference between the charge density of the self-consistent bulk calculation and that of a superposition of respective free-atom charge distributions reveals clearly a charge accumulation near all oxygen centers and charge depletion in the vicinity of vanadium. This confirms the ionic contributions to $\mathrm{V}-\mathrm{O}$ bonding in the bulk oxide. However, a quantification of the ionic charge rearrangement in terms of atom charges is difficult due to the ambiguity of assigning charges to specific atom centers in the FP-LAPW method. A decomposition of the atom charge distribution in $\mathrm{V}_{2} \mathrm{O}_{5}$ based on the Mulliken analysis for a $\mathrm{V}_{10} \mathrm{O}_{49} \mathrm{H}_{18}$ cluster $^{15,22}$ yields $Q(\mathrm{~V})=21.7$ and $Q(\mathrm{O})=8.3$ to 8.7. This seems more reliable despite the inherent problems connected with Mulliken populations.

Earlier cluster calculations on $\mathrm{V}_{2} \mathrm{O}_{5}(010)$ surface properties ${ }^{15,16,27,28}$ were based on an idealized bulk geometry, which differs from the presently optimized and experimental structure as described in Sec. II. Therefore, FPLAPW calculations are performed also for the idealized bulk geometry. A comparison shows that the unit cell total energy of the idealized geometry is higher by only $0.2 \mathrm{eV}$ compared to that of the optimized geometry. Further, the valence band DOS's of the idealized and optimized bulk are very close while the gap between valence and conduction band is re-

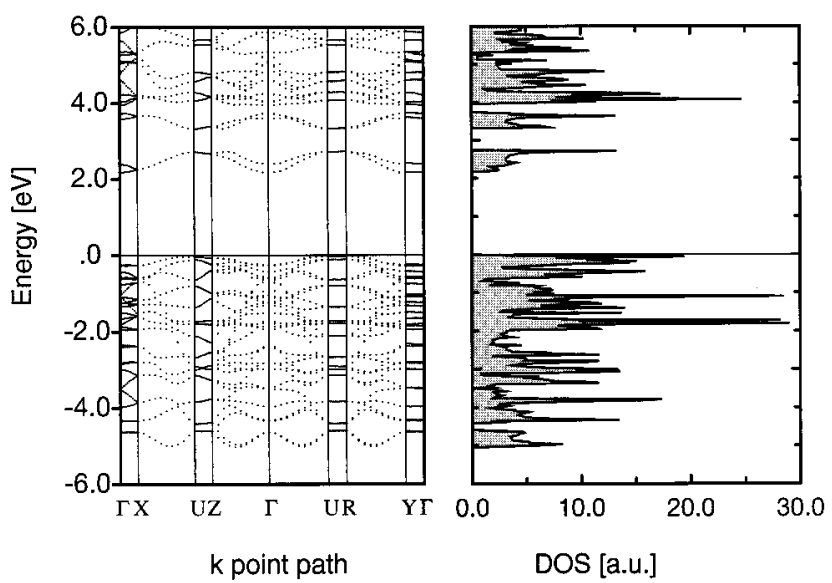

FIG. 6. Band structure and total DOS of a $\mathrm{V}_{2} \mathrm{O}_{5}(010)$ singlelayer slab. The energy bands are shown for characteristic paths connecting high-symmetry points of the irreducible part of the Brillouin zone. For a definition of the high-symmetry points see Fig. 2. All energies $\varepsilon(k)$ are taken with respect to the Fermi energy. The DOS is given in states per unit cell and per eV.

duced from 2.3 (optimized) to $0.8 \mathrm{eV}$ (idealized). This suggests that for adsorption and reactions involving only valence-band states of the substrate, differences between the idealized and optimized bulk geometry are small. In contrast, processes where unoccupied substrate orbitals participate, such as $\mathrm{V} d-d$ transitions will be affected by the choice of the substrate geometry.

\section{$\mathrm{V}_{2} \mathrm{O}_{5}(010)$ single-layer slab calculations}

In order to examine the importance of electronic interlayer coupling in $\mathrm{V}_{2} \mathrm{O}_{5}$ bulk in detail FP-LAPW calculations are carried out on the electronic structure of separated $\mathrm{V}_{2} \mathrm{O}_{5}(010)$ single-layer slabs using the repeated slab geometry. The close similarity between the layer geometry inside the $\mathrm{V}_{2} \mathrm{O}_{5}$ bulk and that of the separated slabs, discussed in Sec. III A, suggests only minor differences in the electronic structure of the two systems. This is confirmed by the numerical results. Figure 6 shows the calculated band structure for a $\mathrm{V}_{2} \mathrm{O}_{5}(010)$ single-layer slab where the left part displays energy bands $\varepsilon(k)$ and the right part presents the corresponding DOS (analogous to the $\mathrm{V}_{2} \mathrm{O}_{5}$ bulk results of Fig. 3). Obviously, the electronic structure of the single-layer slab resembles that of bulk $\mathrm{V}_{2} \mathrm{O}_{5}$ very closely. The valence-band widths $(5.0 \pm 0.5 \mathrm{eV})$, energy gaps $(2.3 \mathrm{eV}$ for direct, $2.1 \mathrm{eV}$ for indirect gap), and even detailed band dispersions agree very well. There are subtle differences in the band structures of the conduction-band region, such as a second split-off pair of bands at $3.5 \mathrm{eV}$ above the Fermi level in the single-layer slab which are, however, not expected to be of great physical significance. Altogether, the present comparison confirms further that electronic interlayer coupling in the bulk material is fairly weak and that the electronic structure at the $\mathrm{V}_{2} \mathrm{O}_{5}(010)$ surface can be modeled reliably by that of a single-layer slab.

Figure 7(a) shows a decomposition of the calculated valence DOS for the $\mathrm{V}_{2} \mathrm{O}_{5}(010)$ single-layer slab into atom projected PDOS contributions. A comparison with the results for bulk $\mathrm{V}_{2} \mathrm{O}_{5}$, shown in Fig. 5, yields the same qualitative 

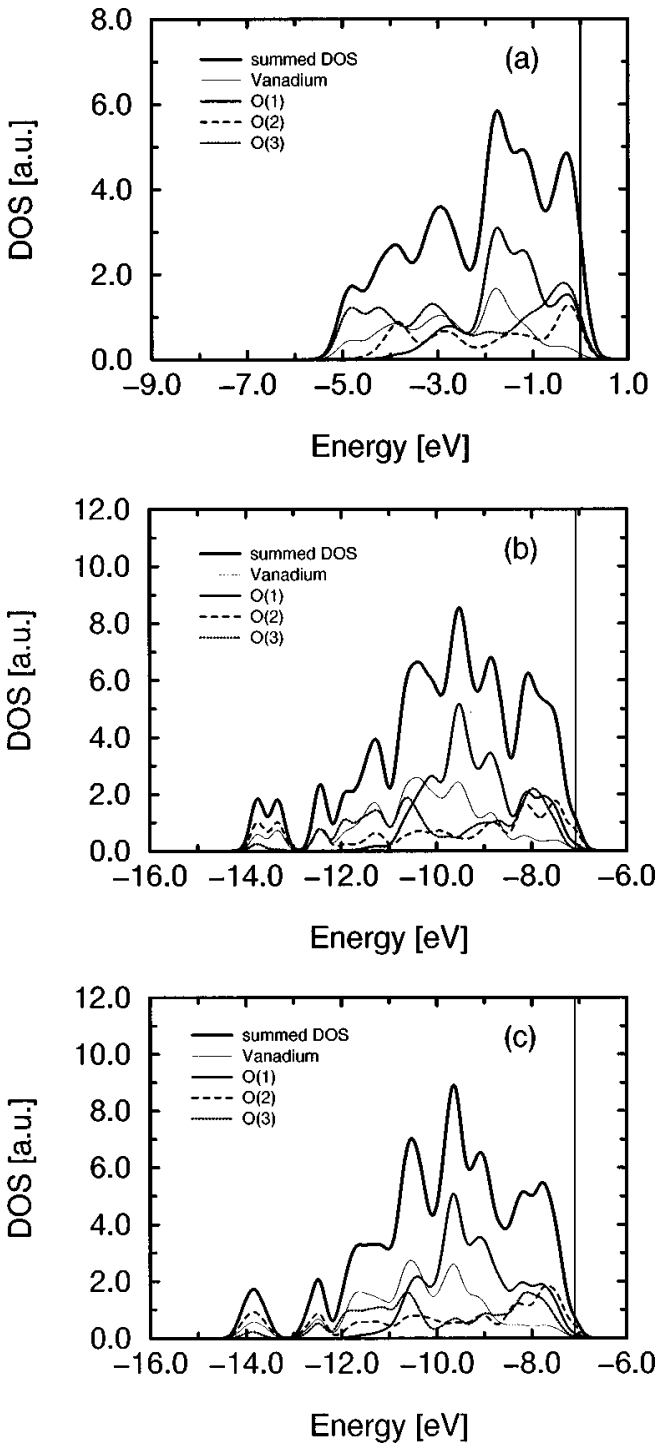

FIG. 7. Atom projected PDOS contributions for (a) the singlelayer slab of $\mathrm{V}_{2} \mathrm{O}_{5}$ in supercell geometry, (b) the $\mathrm{V}_{10} \mathrm{O}_{31} \mathrm{H}_{12}$ cluster, and (c) the $\mathrm{V}_{16} \mathrm{O}_{49} \mathrm{H}_{18}$ cluster. DOS values are given in states per unit cell and per eV. The cluster results were taken from Ref. 22.

results for the PDOS's while their relative weights defining the shapes of the summed DOS's are somewhat different. As in the bulk system, the PDOS due to terminal O(1) $2 p$ states in the slab is peaked between -1 and $-2 \mathrm{eV}$, slightly above the center of the valence region, and is energetically more confined than the PDOS's of the higher coordinated oxygen ions, $\mathrm{O}(2,3)$. An analysis of the relative importance of the different atom contributions shows that the $\mathrm{O}(1)$-derived PDOS is focussed about its maximum more strongly in the layer than in the bulk system. Further, the metal-derived PDOS of the single-layer slab is peaked in the energy region of the maximum of the $\mathrm{O}(1)$-derived PDOS as opposed to the bulk system, which yields a broader distribution. Clearly, these differences reflect the electronic interlayer coupling, which affects vanadyl oxygen ions $\mathrm{O}(1)$ and the underlying vanadium ions more strongly than the other ions of the unit cell. As a result, the summed DOS of the single-layer slab exhibits an energy variation, which resembles the measured photoemission spectrum of the $\mathrm{V}_{2} \mathrm{O}_{5}(010)$ surface, see Fig. 4, more closely than that of the bulk calculation, see Fig. 5.
However, this difference can be partly due to the approximations made in the evaluation of the summed DOS and the neglect of photoemission matrix element effects in the comparison between theory and experiment.

In order to study the local character of binding in the $\mathrm{V}_{2} \mathrm{O}_{5}(010)$ single-layer slab its electronic structure is compared with DFT results from bond-saturated clusters, $\mathrm{V}_{10} \mathrm{O}_{31} \mathrm{H}_{12}$ and $\mathrm{V}_{16} \mathrm{O}_{49} \mathrm{H}_{18}$, representing local environments at the $\mathrm{V}_{2} \mathrm{O}_{5}(010)$ surface. ${ }^{15,22}$ The rather dense distribution of Kohn-Sham valence levels in these clusters allows a meaningful DOS definition by appropriate level broadening. As a result, Figs. 7(b) and 7(c) show decompositions of calculated valence DOS's of $\mathrm{V}_{10} \mathrm{O}_{31} \mathrm{H}_{12}$ and $\mathrm{V}_{16} \mathrm{O}_{49} \mathrm{H}_{18}$ where the atom derived PDOS's are based on Mulliken populations of the different inequivalent atom centers. A comparison of the slab calculations, Fig. 7(a), with those of the clusters, Figs. 7(b) and 7(c), yields very small differences in the valence-band widths and qualitative agreement as to the energy variation of the PDOS's. In all systems, the PDOS due to $2 p$ states of terminal $\mathrm{O}(1)$ is peaked slightly above the center of the valence region and is energetically more focused than the PDOS's of the bridging oxygen ions, $\mathrm{O}(2,3)$. This similarity of the DOS results between the $\mathrm{V}_{2} \mathrm{O}_{5}(010)$ single-layer slab and the clusters suggests strongly that the nature of electronic binding in the oxide and at its surface is quite local. Therefore, local electronic environments at the $\mathrm{V}_{2} \mathrm{O}_{5}(010)$ surface can be described reasonably well by cluster models as large as $\mathrm{V}_{10} \mathrm{O}_{31} \mathrm{H}_{12}$. The cluster DOS's show a separated (double) peak at $-14 \mathrm{eV}$ below vacuum, which is due to $\mathrm{O}-\mathrm{H}$ bonds involving hydrogens used to saturate the cluster periphery. This represents a cluster artifact, which can be neglected for the present purpose.

\section{CONCLUSIONS}

The present theoretical studies provide a rather detailed picture of the geometric and electronic structure of bulk $\mathrm{V}_{2} \mathrm{O}_{5}$ and of its (010) surface. Geometry optimizations of the orthorhombic $\mathrm{V}_{2} \mathrm{O}_{5}$ bulk based on FP-LAPW calculations yield an equilibrium structure, which is in excellent agreement with experimental crystallographic data. ${ }^{23,24}$ The electronic interlayer coupling in bulk $\mathrm{V}_{2} \mathrm{O}_{5}$, which forms a layer crystal, is found to be rather weak. This is evident from a comparison of band structure and DOS results of separated two-dimensional periodic $\mathrm{V}_{2} \mathrm{O}_{5}(010)$ single-layer slabs with those of the bulk. The widths of the valence-band regions as well as the gap energies of the two systems are in good agreement. Further, details of the atom projected PDOS's are very similar as discussed below. This electronic similarity between layer and bulk has implications for geometric properties of the two systems. The present geometry optimization of the single-layer slabs yields atom positions inside the elementary cell, which are very close to those of the bulk. The geometric and electronic similarity between layer and bulk justifies the use of $\mathrm{V}_{2} \mathrm{O}_{5}(010)$ single-layer slabs as meaningful models for the $\mathrm{V}_{2} \mathrm{O}_{5}(010)$ surface. Thus, the present study yields a surface geometry of $\mathrm{V}_{2} \mathrm{O}_{5}(010)$, which is very close to that defined by ideal bulk termination that suggests the absence of major relaxation/reconstruction at the clean single-crystal $\mathrm{V}_{2} \mathrm{O}_{5}(010)$ surface in good agreement with recent experimental results. ${ }^{33,34}$ 
A comparison of the electronic structure of the $\mathrm{V}_{2} \mathrm{O}_{5}(010)$ single-layer slab with that of embedded (bond saturated) surface clusters $\mathrm{V}_{10} \mathrm{O}_{31} \mathrm{H}_{12}$ and $\mathrm{V}_{16} \mathrm{O}_{49} \mathrm{H}_{18}$ (Refs. 15 and 22) can give information about the local character of interatomic binding at the surface. The analysis yields good qualitative agreement between the cluster and slab results concerning atom projected PDOS's of the differently coordinated oxygen ions. This suggests strongly that the nature of electronic binding in the oxide and at its surface is quite local and can thus be described reasonably well by cluster models.

A decomposition of the valence DOS of the single-layer and bulk systems into atom projected PDOS's reveals dominant $\mathrm{O} 2 s p$ contributions. In this analysis the differently coordinated oxygen ions can be identified and are found to contribute to different parts of the valence-energy region. DOS contributions referring to terminal (vanadyl) oxygen, $\mathrm{O}(1)$, are localized near the center of the valence region whereas those of two and three fold bridging oxygen, $\mathrm{O}(2,3)$, yield a broad distribution covering the full valence region and peaking near the periphery. Recent ARUPS experiments for the clean $\mathrm{V}_{2} \mathrm{O}_{5}(010)$ surface ${ }^{41}$ find a three-peak valence spectrum, see Fig. 4, which agrees in its total width with that of the calculated valence bands of the present study. In view of the theoretical DOS results the central peak of the experimental spectrum can be interpreted as being determined by contributions due to vanadyl oxygen while oxygen ions of higher coordination dominate the two peripheral peaks. Altogether, the present comparison of theoretical DOS and experimental ARUPS results for the $\mathrm{V}_{2} \mathrm{O}_{5}(010)$ surface suggests that the different peaks observed in the photoemission experiment may be taken as monitors of the differently coordinated surface oxygen ions. This could be used to study details of catalytic reactions at the oxide surface where surface oxygen ions participate.

\section{ACKNOWLEDGMENTS}

Valuable discussions with B. Tepper, H. Kuhlenbeck, and M. Scheffler are gratefully acknowledged.
*Permanent address: Institute of Catalysis and Surface Chemistry, Polish Academy of Sciences, ul. Niezapominajek, 30-239 Cracow, Poland.

${ }^{1}$ C. N. R. Rao and B. Raven, Transition Metal Oxides (VCH, New York, 1995).

${ }^{2}$ H. K. Kung, in Transition Metal Oxides: Surface Chemistry and Catalysis, edited by B. Delmon and J. T. Yates, Studies in Surface Science and Catalysis, Vol. 45 (Elsevier, Amsterdam, 1989).

${ }^{3}$ V. E. Henrich and P. A. Cox, The Surface Science of Metal Oxides (University Press, Cambridge, 1994).

${ }^{4}$ B. Grzybowska-Swierkosz, Appl. Catal., A 157, 1 (1997), and references therein.

${ }^{5}$ E. E. Chain, Appl. Opt. 30, 2782 (1991), and references therein.

${ }^{6}$ J. Y. Kempf, B. Silvi, A. Dietrich, C. R. A. Catlow, and B. Maigret, Chem. Mater. 5, 641 (1993).

${ }^{7}$ W. Lambrecht, B. Djafari-Rouhani, M. Lannoo, and J. Vennik, J. Phys. C 13, 2485 (1980).

${ }^{8}$ D. W. Bullett, J. Phys. C 13, L595 (1980).

${ }^{9}$ J. C. Parker, D. J. Lam, Y.-N. Xu, and W. Y. Ching, Phys. Rev. B 42, 5289 (1990).

${ }^{10}$ V. Eyert, in Density-Functional Methods: Applications in Chemistry and Materials Science, edited by M. Springborg (Wiley, Chichester, 1997), and references therein.

${ }^{11}$ V. Eyert and K.-H. Höck, Phys. Rev. B 57, 12727 (1998).

${ }^{12}$ J. P. Perdew and Y. Wang, Phys. Rev. B 45, 13244 (1992).

${ }^{13}$ X. Yin, A. Fahmi, A. Endou, R. Miura, I. Gunji, R. Yamauchi, M. Kubo, A. Chatterjee, and A. Miyamoto, Appl. Surf. Sci. 130132, 539 (1998).

${ }^{14}$ R. Ramirez, B. Casal, L. Utrera, and E. Ruiz-Hitzky, J. Phys. Chem. 94, 8960 (1990).

${ }^{15}$ A. Michalak, M. Witko, and K. Hermann, Surf. Sci. 375, 385 (1997).

${ }^{16}$ K. Hermann, A. Michalak, and M. Witko, Catal. Today 32, 321 (1996).

${ }^{17}$ M. Witko, K. Hermann, and R. Tokarz, Catal. Today (to be published).
${ }^{18}$ M. Witko, R. Tokarz, and K. Hermann, Pol. J. Chem. 72, 1565 (1998).

${ }^{19}$ P. Blaha, K.-H. Schwarz, P. Sorantin, and S. B. Trickey, Comput. Phys. Commun. 59, 399 (1990).

${ }^{20}$ B. Kohler, S. Wilke, M. Scheffler, R. Kouba, and C. AmbroschDraxl, Comput. Phys. Commun. 94, 31 (1996).

${ }^{21}$ M. Petersen, F. Wagner, and M. Scheffler (unpublished).

${ }^{22}$ K. Hermann, M. Witko, R. Druzinic, A. Chakrabarti, B. Tepper, M. Elsner, A. Gorschlüter, H. Kuhlenbeck, and H.-J. Freund, J. Electron Spectrosc. Relat. Phenom. 98-99, 245 (1999).

${ }^{23}$ R. W. G. Wyckoff, Crystal Structures, 2nd ed. (Interscience, New York, 1964), Vol. 2.

${ }^{24}$ R. Enjalbert and J. Galy, Acta Crystollogr. Sect. C: Cryst. Struct. Commun. 42, 1467 (1986).

${ }^{25}$ Note that depending on the choice of the orthorhombic crystal axes the layer netplane orientation may also be denoted by (001), interchanging lattice constants $b$ and $c$, as for example in Ref. 23. In this paper, we have adopted an alternative nomenclature taken from Ref. 15 .

${ }^{26}$ X.-G. Wang, W. Weiss, Sh. K. Shaikhutdinov, M. Ritter, M. Petersen, F. Wagner, R. Schlögl, and M. Scheffler, Phys. Rev. Lett. 81, 1038 (1998).

${ }^{27}$ M. Witko, K. Hermann, and R. Tokarz, J. Electron Spectrosc. Relat. Phenom. 69, 89 (1994).

${ }^{28}$ M. Witko and K. Hermann, J. Mol. Catal. 81, 279 (1993).

${ }^{29}$ J. P. Perdew, J. A. Chevary, S. H. Vosko, K. A. Jackson, M. R. Pederson, D. J. Singh, and C. Fiolhais, Phys. Rev. B 46, 6671 (1992).

${ }^{30}$ R. Stumpf and M. Scheffler, Comput. Phys. Commun. 79, 447 (1994).

${ }^{31}$ C. G. Broyden, J. E. Dennis, and J. J. More, J. Inst. Math. Appl. 12, 223 (1973); K. W. Brodlie, The State of the Art in Numerical Analysis, edited by D. A. H. Jacobs (Academic, London, 1977); J. E. Dennis and R. B. Schnabel, Numerical Methods for Unconstrained Optimization and Nonlinear Equations (Prentice-Hall, Englewood Cliffs, NJ, 1983). 
${ }^{32}$ R. Ahuja, O. Eriksson, J. M. Wills, and B. Johansson, Phys. Rev. B 53, 3072 (1996).

${ }^{33}$ R. A. Goschke, K. Vey, M. Maier, U. Walter, E. Göring, M. Klemm, and S. Horn, Surf. Sci. 348, 305 (1996).

${ }^{34}$ A. D. Costa, C. Mathieu, Y. Barbaux, H. Poelman, G. DalmaiVennik, and L. Fiermans, Surf. Sci. 370, 339 (1997).

${ }^{35}$ N. Van Hieu and D. Lichtman, J. Vac. Sci. Technol. 18, 49 (1981).

${ }^{36}$ S. F. Cogan, N. M. Nyugen, S. J. Perrotti, and R. D. Rauh, J. Appl. Phys. 66, 1333 (1989).
${ }^{37}$ A. Z. Moshfegh and A. Ignatiev, Thin Solid Films 198, 251 (1991).

${ }^{38}$ M. Hybertsen and S. G. Louie, Comments Condens. Matter Phys. 13, 5 (1987).

${ }^{39}$ W. Lambrecht, B. Djafari-Rouhani, and J. Vennik, J. Phys. C 14, 4785 (1981).

${ }^{40}$ S. Shin, S. Suga, M. Taniguchi, M. Fujisawa, H. Kanzaki, A. Fujimori, H. Daimon, Y. Ueda, K. Kosuge, and S. Kachi, Phys. Rev. B 41, 4993 (1990).

${ }^{41} \mathrm{~B}$. Tepper (private communication). 